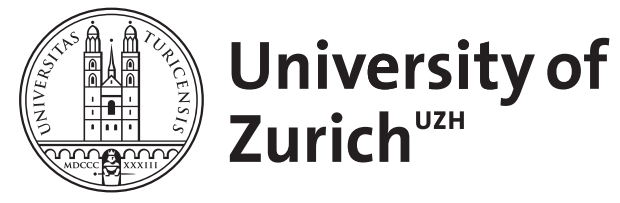

Zurich Open Repository and Archive

University of Zurich

University Library

Strickhofstrasse 39

CH-8057 Zurich

www.zora.uzh.ch

Year: 1994

\title{
Nikolaus von Kues und der Dialog mit dem Islam
}

Rudolph, Ulrich

DOI: https://doi.org/10.1515/islm.1994.71.1.95

Posted at the Zurich Open Repository and Archive, University of Zurich

ZORA URL: https://doi.org/10.5167/uzh-80152

Journal Article

Originally published at:

Rudolph, Ulrich (1994). Nikolaus von Kues und der Dialog mit dem Islam. Der Islam, 71:95-108.

DOI: https://doi.org/10.1515/islm.1994.71.1.95 


\title{
Nikolaus von Kues und der Dialog mit dem Islam
}

\author{
Ulrich Rudolph (Göttingen)
}

Die Auseinandersetzung mit dem Islam gehört zu den Themen, die Europa während des gesamten Mittelalters bewegt und begleitet haben. Seit der Eroberung Spaniens im 8. Jahrhundert und, mehr noch, seit der Einnahme Siziliens waren Muslime als Herrscher und als Herausforderung im Abendland präsent. Und daran sollte sich, solange die Epoche währte, nichts ändern. Denn als Sizilien längst zurückgewonnen war und im 15. Jahrhundert selbst die iberische Halbinsel im Begriff stand, wieder ein christliches Bollwerk zu werden, sah sich der Balkan einem osmanischen Siegeszug gegenüber, der eindrucksvoller und beängstigender als jede frühere muslimische Eroberung in Europa voranschritt.

Das Problem Islam bildete mithin eine Konstante in der mittelalterlichen Geschichte und zwang die Europäer immer wieder, zu reagieren und Stellung zu beziehen. ${ }^{1}$ ) Aber so beständig diese Herausforderung an das

1) Das Islam-Bild des europäischen Mittelalters wurde inzwischen in einer Reihe von grundlegenden Studien behandelt. Vgl. vor allem U. Monneret de Villard, Lo studio dell'Islam in Europa nel XII e nel XIII secolo (Studi e testi 110), Città del Vaticano 1944; M. Th. d'Alverny, La connaissance de l'Islam en Occident du IX siècle au milieu du $\mathrm{XII}^{\mathrm{e}}$ siècle, in: L'Occidente e l'Islam nell'alto medioevo II (Settimane di studio del centro Italiano di studi sull'alto medioevo XII), Spoleto 1965, S. 577-602; N. Daniel, Islam and the West. The Making of an Image, Edinburgh $1966^{3}$; R. W. Southern, Western Views of Islam in the Middle Ages, Cambridge/ Mass. $1978^{2}$ (dt. Übers.: Das Islambild des Mittelalters, Stuttgart u.a. 1981). M. Rodinson, Die Faszination des Islam, München $1991^{2}$ (nach der frz. OriginalAusgabe, Paris 1980) baut auf diesen Untersuchungen äuf und verfolgt das europäische Islam-Bild bis in die Gegenwart, ebenso.J. D. J. Waardenburg in dem kürzlich erschienenen Artikel Mustashrikūn, in: $\mathrm{EI}^{2}$ VII 735-753, wobei sein Interesse insbesondere der Sichtweise der Orientwissenschaft gilt. Immer noch wichtig ist auch die Monographie von J. Fück, Die arabischen Studien in Europa bis in den Anfang des 20. Jahrhunderts, Leipzig 1955, die ausführliche Kapitel über die Sprachkenntnisse des Mittelalters enthält. - Zum heutigen Stand der Forschung vgl. G. C. Ana- 
Abendland auch gewesen sein mag - sie hat doch nicht immer zu den gleichen Reaktionen geführt. Vielmehr fällt auf, daß die Antworten auf die Bedrohung wechselten und daß man stets neue Wege suchte, um des alten Problemes Herr zu werden. ${ }^{2}$ ) Meistens konzentrierten sich die Hoffnungen in Europa auf die bewaffnete Auseinandersetzung ${ }^{3}$ ), manchmal.verlagerten sie sich auf die literarische Polemik ${ }^{4}$ ); und eine Zeitlang favorisierte man

wati, Islam et Christianisme: La rencontre de deux cultures en Occident au Moyen Age, in: Mịdeo 20/1991/233-299; zur Auseinandersetzung der Byzantiner mit dem Islam vgl. die zahlreichen Arbeiten von A. Th. Khoury, so etwa Apologétique byzantine contre l'Islam (VIII'-XIII'), Altenberge 1982. - Einen bibliographischen Überblick zum Thema gibt L. Hagemann, Zur Auseinandersetzung des Christentums mit dem Islam im Mittelalter und in der Reformationszeit, in: Verkündigung und Forschung 32/1987/43-62.

2) Der erste Versuch, die verschiedenen Etappen zu unterscheiden und zu benennen, stammt wohl von Southern, Western Views. Er trennt „The Age of Ignorance" (von 700 bis 1140) von "The Century of Reason and Hope" (von 1140 bis zum Ende des 13. Jahrhunderts) sowie von „The Moment of Vision“, den er in der Mitte des 15. Jahrhunderts erkennen will. Rodinson, Faszination, variiert diese Einteilung. Seine erste Etappe („Das Mittelalter: Kampf zweier Welten“) dauert bis zum Ende des 12. Jahrhunderts; die zweite („Ein weniger polemisches Bild entsteht und vergeht“) umfaßt das 13. Jh.; die dritte („Die engere Koexistenz: Der Feind wird zum Partner ${ }^{\circ}$ ) setzt mit dem Ende des 14. Jhs. ein. Interessant sind in diesem Zusammenhang auch die Überlegungen von C. Colpe, Historische und theologische Gründe für die abendländische Angst vor dem Islam, in: ders., Problem Islam, Frankfurt a. M. 1989, S. 11-38. Colpe geht es allerdings nicht so sehr um die genaue Datierung bestimmter Islambilder, sondern um deren typologische Einordnung.

${ }^{3}$ ) Sie begann im frühen 8. Jh. mit den Kämpfen in Spanien, dürfte aber lange Zeit im Bewußtsein weiter Teile Europas keine allzu große Rolle gespielt haben (dazu Southern, Western Views, S. $14 \mathrm{ff}$.). Das änderte sich erst mit den Kreuzzügen, also gegen Ende des 11. Jhs.

4) In der griechischen Tradition hat die ernsthafte literarische Auseinandersetzung eine lange Geschichte (beginnend mit Johannes Damascenus), im lateinischen Westen setzte sie erst Mitte des 12. Jhs. ein. Der Initiator war hier Petrus Venerabilis, der den Koran ins Lateinische übertragen ließ und sich selbst mit der Widerlegung des Islams befaßte. Zu ihm vgl. J. Kritzeck, Peter the Venerable and Islam, Princeton 1964 und R. Glei (ed.), Petrus Venerabilis - Schriften zum Islam, Altenberge 1985 (Corpus Islamo-Christianum. Series Latina. 1). Einen Überblick über das Schrifttum gibt L. Hagemann, Bibliographie du dialogue islamo-chrétien. Auteurs chrétiens de langue latine des $\mathrm{XI}^{e}$ et $\mathrm{XII}^{e}$ siècles, in: Islamochristiana 5/ 1979/312-316 bzw. Autéurs chrétiens du monde latin des $\mathrm{XIII}^{\mathrm{e}}$ et $\mathrm{XIV}^{e}$ siècles, in: Islamochristiana 6/1980/260-278. 
das Projekt der Mission ${ }^{5}$ ), um nur die bekanntesten Versuche zu nennen, die während des Mittelalters unternommen worden sind. Doch sie alle schlugen fehl oder erreichten zumindest nicht, was man sich von ihnen versprochen hatte. So kam es, daß gegen Ende der Epoche noch einmal eine Idee von ganz anderer Art vorgetragen worden ist.

Sie wurde laut im Jahre 1454, also kurze Zeit, nachdem Konstantinopel in die Hände der Türken gefallen war. Aber der Vorschlag klingt in unseren heutigen Ohren überraschend modern und vertraut. Er lautete nämlich, man möge eine Konferenz abhalten, auf der sich die Vertreter der verschiedenen Religionen begegnen sollten. Dort könnten sie über die Wahrheiten ihres Glaubens disputieren. Und selbst wenn sie sich nach Jahren des Verhandelns ergebnislos trennen sollten, hieß es, richte ein solches Gespräch doch keinen Schaden an und sei deswegen immer einem Krieg vorzuziehen.

Die ungewöhnliche Idee stammte von einem Spanier ${ }^{6}$ ), aber sie wurde von niemandem so unterstiutzt wie von Nikolaus von Kues. Denn Cusanus begrüßte nicht nur den Vorschlag. Er machte sich auch Gedanken über eine

5) Der Missionsgedanke war ein Privileg des 13. Jhs. Vgl. B. Altaner, Die Dominikanermission des 13. Jahrhunderts, Habelschwerdt 1924; M. Grabmann, Die Missionsidee der Dominikanertheologen des 13. Jahrhunderts, in: Zeitschrift für Missions- und Religionswissenschaft 1/1911/137-146; D. Berg, Kreuzzugsbewegung und Propagatio fidei. Das Problem der Fransziskanermission im 13. Jahrhundert und das Bild von der islamischen Welt in der zeitgenössischen Ordenshistoriographie, in: A. Zimmermann/I. Craemer-Ruegenberg (Hg.), Orientalische Kultur und europäisches Mittelalter (Miscellanea Mediaevalia. 17), Berlin/New York 1985, S. 59-76. Selbst Thomas v. Aquin schrieb seine berühmte Summa contra gentiles im Dienste der Mission, ebenso die kleine Schrift De rationibus fidei, die neuerdings in einer kommentierten zweisprachigen Ausgabe von L. Hagemann/ R. Glei, Altenberge 1987 (Corpus Islamo-Christianum. Series Latina. 2), vorliegt.

$\left.{ }^{6}\right)$ Nämlich von Johannes von Segovia, der seinen Vorschlag in einer umfangreichen Korrespondenz drei wichtigen Repräsentanten der Kirche unterbreitete: Nikolaus von Kues; Jean Germain, Bischof von Châlon-sur-Saône; und Enea Silvio Piccolomini, der als Papst Pius II. bekannt geworden ist. Der Briefwechsel wurde häufig in der Sekundärliteratur beschrieben und gilt, seit seine Bedeutung von Southern betont wurde, geradezu als ein Kristallisationspunkt der verschiedenen europäischen Reaktionen auf den Fall von Konstantinopel. Man wird aber doch fragen müssen, ob seine Aussagekraft damit nicht überbewertet worden ist. Vgl. zu den näheren Umständen R. Haubst, Johannes von Segovia im Gespräch mit Nikolaus von Kues und Jean Germain über die göttliche Dreieinigkeit und ihre Verkündigung vor den Mohammedanern, in: Münchener Theologische Zeitschrift 2/1951/115129; Klibansky/Bascour in der Praefatio (p. XLVff.) zu ihrer Edition von Cusanus' De pace fidei (vgl. u. Anm. 16); D. Cabanelas Rodriguez, Juan de Segovia y el problema islámico, Madrid 1952; Southern, Western Views, S. 85 ff.; Rodinson, Faszination, S. 50f.; Heinemann, Einheit in Verschiedenheit (vgl- u. Anm. 20), S. 27 ff. 
mögliche Verwirklichung ${ }^{7}$ ), obwohl es dazu leider niemals gekommen ist. Damit hat sich der Kardinal, so scheint es, einen Platz in der Geschichte des religiösen Dialoges gesichert, und deswegen kann es auch nicht verwundern, wenn gerade in jüngster Zeit diese Seite seines. Denkens ganz besonders betont worden ist. ${ }^{8}$ ) Cusanus gilt heute nicht nur als der kühne Verfechter der docta ignorantia und der coincidentia oppositorum. Ihm wird ebenfalls zugeschrieben, sich in großartiger Weise um einen „Brücken-

${ }^{7}$ ) So schlägt Cusanus zum Beispiel vor, christliche Kaufleute aus Kairo, Alexandria, Armenien und Griechenland zusammenzurufen, damit sie aus erster Hand über die Ideen und Gebräuche der Sarazenen berichten können. Oder er weist darauf hin, daß man als Unterhändler bei den Gesprächen eher weltliche Fürsten als Priester einsetzen solle, weil sie bei den Türken in höherem Ansehen stünden. Vgl. dazu seine Epistula ad Ioannem de Segobia, die von Klibansky/Bascour im Anhang an die Edition von De pace fidei herausgegeben worden ist (bes. S. 97, $12 \mathrm{ff}$.).

${ }^{8}$ ) Die Literatur zu Cusanus' Auseinandersetzung mit dem Islam hat in den letzten Jahrzehnten einen erstaunlichen Umfang erreicht. Sie umfaßt nicht nur zwei Monographien: F. H. Burgevin, Cribatio Alchorani. Nicholas Cusanus's Criticism of the Koran in the Light of His Philosophy of Religion, New York u.a. 1969 und L. Hagemann, Der Kur'ān in Verständnis und Kritik bei Nikolaus von Kues. Ein Beitrag zur Erhellung islamisch-christlicher Geschichte, Frankfurt a.M. 1976 (Frankfurter Theologische Studien. 21). Zu ihr zählt auch eine ganze Reihe von Aufsätzen, die aus sehr unterschiedlichen Perspektiven geschrieben worden sind: G. Hölscher, Nikolaus von Cues und der Islam, in: Zeitschrift für philosophische Forschung 2/1947/259-274; N. Rescher, Nicholas of Cusa on the Qur'ān. A fifteenthcentury encounter with Islam, in: Muslim World 55/1965/195-202, wieder abgedruckt in: ders., Studies in Arabic Philosophy, Pittsburgh 1967, S. 137-146; G. C. Anawati, Nicolas de Cues et le problème de l'Islam, in: Nicolò Cusano agli inizi del mondo moderno. Atti del Congresso internazionale in occasione del $\mathrm{V}$ centenario della morte di Nicolò Cusano, Bressanone, 6-10 settembre 1964, Florenz 1970 (Pubblicazioni della Facoltà di Magistero dell'Università di Padova. 12), S. 141173 ; S. Raeder, Der Christus des Korans in der Sicht des Nikolaus von Kues, in: Christentum und Islam 6/1975/71-93; J. E. Biechler, Nicholas of Cusa and Muhammad: A fifteenth-century encounter, in: Downside Review 101/1983/50-59; L. Hagemann, Nikolaus von Kues im Gespräch mit dem Islam, Altenberge 1983 (Christlich-Islamisches Institut. Abhandlungen. 1). - Vollends im Mittelpunkt stand das Thema der religiösen Verständigung dann bei zwei Tagungen, deren Akten in den Mitteilungen und Forschungsbeiträgen der Cusanus-Gesellschaft (im folgenden zitiert als: MFCG) veröffentlicht worden sind: Nikolaus von Kues als Promotor der Ökumene. Akten des Symposions in Bernkastel-Kues vom 22. bis 24. September 1970, hg. von R. Haubst, Mainz 1971 (MFCG 9) und: Der Friede unter den Religionen nach Nikolaus von Kues. Akten des Symposions in Trier vom 13. bis 15. Oktober 1982, hg. von R. Haubst, Mainz 1984 (MFCG 16). 
schlag“9) zum Islam bemüht zu haben, ja, überhaupt „der kühnste aller Ökumeniker ${ }^{« 10}$ ) gewesen zu sein.

Allerdings ist diese Einschätzung keineswegs unumstritten $\left.{ }^{11}\right)$, und man wird auch nicht zuviel wagen, wenn man behauptet, daß die religionsphilosophischen Gedanken des Cusanus noch nicht mit all ihren Implikationen diskutiert worden sind. ${ }^{12}$ ) Daher soll im folgenden noch einmal der Versuch

$\left.{ }^{9}\right)$ R. Haubst in: MFCG 9/1971/87 Anm. 17.

${ }^{10}$ ) B. Hanssler in: MFCG 9/1971/190.

11) Insgesamt überwiegt in der Forschung die Tendenz, Cusanus' Auseinandersetzung mit dem Islam positiv zu bewerten. So schreibt Rescher, Nicholas of Cusa, S. 146: „As Christianity and Islam draw increasingly into peaceful contact with one another in our own time, Nicholas of Cusa deserves more and more clearly to be numbered as a member of the small band of men of prophetic insight." A. Schall, in: MFCG 9/1971/87: „Es wird immer noch behauptet, das Mittelalter hätte sich nur in einem wilden, bösen Kampf gegen den Islam betätigt. Daß eine so positive Stellungnahme vorgelegen hat wie in der Cribratio Alchoran, ist offensichtlich bisher kaum durchgedrungen. “ Hagemann, Kur’ān, S. 3: „Ist es doch Nikolaus von Kues, dem in der Geschichte des Ökumenismus oder dessen Vorbereitung zweifellos der erste Platz zukommt." Biechler, Nicholas of Cusa, S. 58: „Evaluated alongside the thought of other theologians, Nicholas of Cusa's intellectual encounter with Muhammad and Islam ... . stands out in dramatic and positive contrast“. K. Kremer, in: MFCG 16/1984/158: „Cusanus hat sich aufgemacht, ... um die jedem Glauben gemeinsam zugrundeliegenden Voraussetzungen aufzudecken. Dafür bietet er den ganzen Genius seiner spekulativen Befähigung auf ...". Rodinson, Faszination, S. 51: „. . . Nikolaus von Kues, . . . der mit seiner Cribratio Alcorani (1460) versuchte, eine präzise philologische und historische Untersuchung des Korans vorzunehmen." - Allerdings gab es auch kritische Einwände, die vielleicht am deutlichsten von Anawati, Nicolas de Cues, S. 172 formuliert worden sind: „Nicolas de Cues peut-il nous être utile pour le dialogue islamo-chrétien? Au risque de déplaire aux admirateurs sans réserve du célèbre Cardinal, je répondrai sans hésitation: Non“. Anawati ist denn auch prompt für seine Haltung getadelt worden, zunächst von Haubst, in: MFCG 9/1971/87 Anm. 17 und im Anschluß daran von Hagemann, Kur'ān, S. 184. Aber er steht mit seiner nüchternen Betrachtungsweise keineswegs allein. So schreibt Southern, Western Views, S. 94: „I do not suppose that this analysis of the text of the Koran has any value now . . . . Daniel, Islam, S. 278: „Here was a fresh mind working over old themes with varying success. “ Burgevin, Cribratio, S. 115: „As we have indicated, the Cribratio shows a concern for unity, an Evangelical zeal, rather than tolerance“. M. Gandillac, in: MFCG 16/1984/201: „Bei aller wissenschaftlichen Neugierde war Nikolaus indes außerstande, auch mit den ihm fremden Religionen als solchen ein ernstes Vergleichen zu entwickeln . . .“. Im übrigen sieht auch Hagemann Cusanus inzwischen kritischer, wie sein vorsichtiges Fazit in dem Beitrag Nikolaus von Kues im Gespräch mit dem Islạm, S. 26 f. zeigt.

12) Die religionsphilosophischen Aspekte wurden bịslang von $\mathrm{K}$. Kremer, Die Hinführung (manuductio) von Polytheisten zum Einen, von J'uden und Mohamme- 
unternommen werden, seine Auseinandersetzung mit dem Islam zu beleuchten. Dabei ist es zunächst nötig, seinen Ausgangspunkt zu beschreiben und dann zu verfolgen, welches Bild er von der anderen Religion entworfen hat. Aber das eigentliche Ziel unserer Überlegungen ist eine Bewertung: nämlich zu antworten auf die Frage, ob der Kardinal tatsächlich auf die Muslime zuging und ob er ihnen ein auch heute noch bedenkenswertes Angebot zum Dialog unterbreitete.

\section{II}

Daß Nikolaus von Kues für den Vorschlag einer Konferenz plädierte, hat seine Zeitgenossen vermutlich nicht überrascht. Denn gerade ihn zeichneten mehrere Eigenschaften aus, die seine Person als prädestiniert für ein solches Vorhaben erscheinen ließen. Er hatte den Islam seit Jahren theoretisch studiert. ${ }^{13}$ ) Er besaß praktische Erfahrungen mit Glaubensgesprächen, da er sowohl an den Verhandlungen mit der oströmischen Kirche $^{14}$ ), als auch an der Diskussion mit den Hussiten ${ }^{15}$ ) beteiligt gewesen war. Überdies stammte von ihm eine Schrift, die unzweifelhaft seinen Willen zum Frieden dokumentierte. Gemeint ist sein berühmtes Werk „Über den Frieden im Glauben" (De pace fidei), in dem das Konzept einer universalen religiösen Verständigung entwickelt wird. ${ }^{16}$ )

danern zum Dreieinen Gott, in: MFCG 16/1984/126-163 und vor allem von Burgevin, Cribratio behàndelt. Burgevin konzentriert sich allerdings sehr darauf, Cusanus' Explikation der christlichen Glaubenswahrheiten zu beschreiben. Zudem geht er von einem historischen Vergleich zwischen der türkischen Bedrohung im 15. Jh. und dem russischen Expansionsdrang im 20. Jh. aus (Cribratio, S. $112 \mathrm{f}$.), den man in dieser-Form nicht teilen wird.

${ }^{13}$ ) Spätestens seit 1437, als Cusanus im Auftrag des Papstes nach Konstantinopel reiste, dürfte sein Interesse für den Islam groß gewesen sein. Vgl. dazu seine eigenen Worte im ersten Prolog der Cribratio, N. 2. Dort (N. 2 u. 4) nennt er auch die Quellen, auf die er bei seinen Studien zurückgegriffen hat. Dazu im einzelnen Hagemann, Kur’ān, S. 15 ff.

14) W. Krämer, Der Beitrag des Nikolaus von Kues zum Unionskonzil mit der Ostkirche, in: MFCG 9/1971/34-52.

15) H. Hallauer, Das Glaubensgespräch mit den Hussiten, in: MFCG 9/1971/ 53-75.

${ }^{16}$ ) Die maßgebliche Edition des Textes stammt von R. Klibansky/H. Bascour, Nicolai de Cusa Opera omnia. VII: De pace fidei, Hamburg $1970^{2}$. Übersetzungen gibt es zahlreiche, so auf.Deutsch: L. Mohler, Über den Frieden im Glauben, Leipzig 1942 (NvKdÜ. 8); D. und W. Dupré, Nikolaus von Kues. Philosophisch-theologische Schriften, III, Wien 1967, S. 705-797; R. Haubst, Nikolaus von Kues. Textaus- 
De pace fidei war im Sommer 1453 entstanden. ${ }^{17}$ ) Damals stand Cusanus unter dem unmittelbaren Eindruck der Eroberung von Konstantinopel und versuchte, ein Zeichen zu setzen gegen die Kreuzzugshysterie, die in Europa allenthalben um sich griff. ${ }^{18}$ ) Am Anfang seines Textes heißt es ausdrücklich, man solle aufhören, im Namen der Religionen einander Greueltaten anzutun. ${ }^{19}$ ) Und dann schildert Nikolaus einen fiktiven Dialog, an dem Vertreter aller großen Nationen und Glaubensgemeinschaften teilnehmen und einen Konsens über die Grundwahrheiten der Religionen erzielen. ${ }^{20}$ )

Der Text enthielt ohne $Z$ weifel eine Vision, in die auch der Islam ganz bewußt eingeschlossen sein sollte. Aber Cusanus war viel zu sehr ein Mann des Handelns und der Politik, um nicht zu wissen, daß eine Annäherung zwischen den Religionen nicht allein mit solchen visionären Schriften zu erreichen ist. Deswegen sehen wir ihn in den Jahren nach 1453 unentwegt auf der Suche nach praktischen Möglichkeiten der Begegnung. Dabei war sich der Kardinal des Weges, den man einzuschlagen hatte, nicht ganz sicher, denn er bezog auch ältere, längst bekannte Antworten auf den Islam in seine Überlegungen mit ein. So griff er etwa zurück auf das Mittel der Polemik und bat einen Schüler, Muhammads Irrlehren in einer Kampfschrift zu widerlegen. ${ }^{21}$ ) Zudem schloß er die Option, daß man womöglich doch einen Kreuzzug gegen die Türken führen müsse, niemals grundsätzlich aus. ${ }^{22}$ ) Aber sein eigentliches Anliegen blieb das Gespräch. Und um die

wahl in deutscher Übersetzung. 1: De pace fidei. Der Friede im Glauben, Trier 1982. Auf Französisch zuletzt: R. Galibois, Nicolas de Cues, La paix de la foix, Sherbrooke 1977. Auf Englisch: J. E. Biechler/H. L. Bond, Nicholas of Cusa on Interreligious Harmony. Text, Concordance and Translation of De pace fidei, Lewiston u. a. 1990.

$\left.{ }^{17}\right)$ Vgl. Klibansky/Bascour, Praefatio zur Edition, p. IXff.

${ }^{18}$ ) Über die abendländische Reaktion auf den Fall von Byzanz informieren A. Pertusi, La caduta di Constantinopoli. Le testimonianze dei contemporanei, Mailand 1976; E. Meuthen, Der Fall von Konstantinopel und der lateinische Westen, in: MFCG 16/1984/15-30.

$\left.{ }^{19}\right)$ De pace fidei I 1, S. 3,3-7.

$\left.{ }^{20}\right)$ Den Text beleuchten die verschiedenen Beiträge in: MFCG 16/1984. Vgl. daneben M. Seidlmayer, „Una religio in rituum varietate“. Zur Religionsauffassung des Nikolaus von Kues, in: Archiv für Kulturgeschichte 36/1954/145-207; W. Heinemann, Einheit in Verschiedenheit. Das Konzept eines intellektuellen Religionenfriedens in der Schrift „De pace fidei“ des Nikolaus von Kues, Altenberge 1987.

${ }^{21}$ ) So entstand der Text Contra perfidiam Mahometi des Dionysius Cartusianus; dazu Hagemann, Kur’ān, S. 50 ff.

22) Einen Kreuzzug zu führen, war vor allem das ehrgeizige Projekt Papst Pius II., in dessen Auftrag sich auch Cusanus dafuir einsetzen mußte. Er starb sogar am 11. August 1464 in Todi bei den Vorbereitungen zu diesem (nie zustandegekommenen) Unternehmen. Vgl. dazu E. Meuthen, Die letzten Jahre des Nikolaus von Kues. Biographische Untersuchungen nach neuen Quellen, Köln/Opladen 1958. 
Christen auf diese Möglichkeit vorzubereiten, genauer: um ihnen dafür die nötigen Argumente an die Hand zu geben, verfaßte Nikolaus im Winter 1460/61 ein Buch mit dem Titel "Sichtung des Korans" (Cribratio Alkorani), das für uns höchstes Interesse besitzt. ${ }^{23}$ ) In ihm äußerte er sich ausführlich zu Muhammad und zur heiligen Schrift der Muslime, so daß wir an diesem Text sehr präzise verfolgen können, von welchen Vorstellungen der Kardinal bei seinen Bemühungen um einen Dialog ausgegangen ist.

\section{III}

Studiert man die Cribratio etwas genauer, so stellt man bald fest, daß ihr Autor den Islam aus unterschiedlichen Perspektiven betrachtet. Er entwirft vor dem Leser keineswegs ein festes, für immer gültiges Bild, sondern setzt im Verlaufe des Textes mehrfach neu an, um seine Sicht der muslimischen Religion zu erklären. ${ }^{24}$ ) Dabei ist der erste dieser Versuche vielleicht schon der originellste. Denn in ihm löst sich Cusanus am meisten von den früheren Deutungen des Mittelalters und vertraut mehr als sonst auf seine eigene Philosophie.

Alle Menschen, so heißt es da nämlich, trügen in sich ein Streben nach Gott, dem Einen und höchsten Guten. Aber als Menschen sei ihnen, wie jeder wisse, die wahre Erkenntnis Gottes versperrt. Deswegen seien auch schon viele aufgetreten, um uns den Weg zum Heil zu weisen, darunter Moses, Jesus und Muhammad, denen man „die namhafteren Beschreibungen des besagten Weges" (magis famosae descriptiones dictae viae) verdanke. Aber nur Jesus könne die Wahrheit tatsächlich gewußt haben. Denn er war „die allwissende göttliche Weisheit" (omnisciens divina sapientia),

${ }^{23}$ ) Kritische Edition von L. Hagemann, Nicolai de Cusa Opera omnia. VIII: Cribratio Alkorani, Hamburg 1986. Die erste deutsche Übersetzung stammt aus den vierziger Jahren: Nikolaus von Cues, Sichtung des Alkorans. Erstes. Buch von P. Naumann mit Anmerkungen von G. Hölscher, Leipzig $1948^{2}$ (NvKdÜ. 6. Philosophische Bibliothek. 221); Zweites und Drittes Buch von G. Hölscher, Leipzig 1946 (NvKdÜ. 7. Philosophische Bibliothek. 222). Zur Zeit wird eine lateinisch-deutsche Parallelausgabe von L. Hagemann/R. Glei erstellt, in der die ersten beiden Büicher der Cribratio bereits erschienen sind: Nikolaus von Kues, Sichtung des Korans. Erstes Buch, Hamburg 1989 (NvKdÜ. 20a. Philosophische Bibliothek. 420 a); Zweites Buch, Hamburg 1990 (NvKdÜ. 20 b. Philosophische Bibliothek. 420 b). Nach dieser Ausgabe wird der Text der Cribratio im folgenden auf Deutsch zitiert. Für die Literatur zu dieser Schrift vgl. oben Anm. 8.

${ }^{24}$ ) Für eine knappe Inhaltsübersicht des ganzen Werkes vgl. immer noch Anawati, Nicolas de Cues, S. $153 \mathrm{ff}$. 
Moses und Muhammad dagegen bloße Menschen - und damit per Definition außerstande, der Erkenntnis des Göttlichen wirklich teilhaftig zu sein. ${ }^{25}$ )

Die Überlegung ist delikat und hat verschiedene interessante Aspekte. Für unseren Zusammenhang besitzt jedoch nur Bedeutung, wie hier Muhammad beurteilt wird. Von ihm heißt es, er habe die Wahrheit nicht wissen können. Aber es wird auch erklärt, er habe die Wahrheit aufrichtig gesucht, und das ist der erste wichtige Punkt, den es für uns festzuhalten gilt.

Doch, wie gesagt: Cusanus bleibt bei dieser einen Überlegung nicht stehen, sondern hebt gleich zu einer zweiten Darstellung an, bei der die Dinge anders gewichtet werden. Jetzt berichtet er uns Einzelheiten aus dem Leben des Propheten, wobei seine Ausführungen diesmal nicht originell, sondern zutiefst der älteren mittelalterlichen Polemik verpflichtet sind. Muhammad, so hören wir nun, sei in Mekka von einem Mönch namens Sergius unterrichtet worden. Dieser habe ihm das Christentum in der nestorianischen Form gepredigt, und der Schüler Muhammad sei der neuen Botschaft zunächst auch bereitwillig gefolgt. Indessen: Drei äußerst listige Juden (tres astutissimi Iudaei) hätten es verstanden, ihn wieder vom wahren Glauben abzubringen. Und sie hätten auch dafür Sorge getragen, daß nach seinem Tode der Koran verändert und verfälscht worden sei. ${ }^{26}$ )

Damit sind wir bei der Biographie des Propheten angekommen, oder besser: bei einer bekannten mittelalterlichen Legende, die Cusanus hier nur wiedergibt. Aber die Übernahme solcher desavouierender Vorstellungen hat doch Folgen, denn sie verändert nachhaltig das zuvor beschriebene Bild. Muhammad gilt jetzt nicht mehr als der aufrechte Wahrheitssucher. Er hat die Wahrheit sogar berührt, doch, was noch wichtiger ist, er hat sie später wieder verloren. Damit verdunkelt sich sein Porträt, weil es Züge der Einfalt und der Unselbständigkeit gewinnt. Folglich ist es kein Wunder, wenn Cusanus nun auch harte Worte für den muslimischen Propheten findet. So lautet ein Vorwurf, er könne das Christentum nur aufgegeben haben aus Unwissenheit (ignorantia) oder aus böser Absicht (perversitas intentionis) oder aber, weil er seinen eigenen Rubm suchte (sui ipsius gloriam quaesivit) ${ }^{27}$ ) Und vom Koran heißt es, er könne unter diesen Umständen nicht

${ }^{25}$ ) Cribratio, Prologus N. 5,1-9,5; zur Beschreibung des Prologes vgl. Anawati, Nicolas de Cues, S. 153-7; zu einem ähnlichen philosophischen Ansatz in De pace fidei vgl. Kremer, Die Hinführung, in: MFCG 16/1984/127ff.

${ }^{26}$ ) Cribratio, Alius Prologus; vgl. Anawati, Nicolas de Cues, S. 157-160; zu Herkunft und Tradition der Legende ausführlich Hagemann; Kur’ān, S. 74 ff. sowie ders., Nikolaus von Kues im Gespräch, S. $10 \mathrm{ff}$.

${ }^{27}$ ) Cribratio, Prologus, N. 9,5-9. 
als Gottes Wort gelten. Sein Urheber sei wohl eher „der Fürst dieser Welt“ (princeps huius mundi), also niemand anderes als der Teufel. ${ }^{28}$ )

Man sollte denken, mit diesem Satz sei ein definitives Urteil gesprochen und der Leser wisse endgültig, was er vom İslam zu halten habe. Indes: Selbst dieses neue düstere Bild gilt nur bedingt. Denn Cusanus überrascht uns in einem späteren Kapitel ein weiteres Mal, indem er seiner Interpretation noch eine letzte Facette hinzufügt. Sie macht aus dem ruhmsüchtigen Ignoranten Muhammad plötzlich wieder eine Figur im göttlichen Heilsplan, und das wird möglich, indem die Vorgaben der gerade beschriebenen Legende eine radikale Umdeutung finden. Denn, so lautet diese dritte Überlegung: Muhammad kannte nicht nur das Christentum. Er kannte auch seine ungebildeten arabischen Landsleute (rudes Arabes) und wußte genau, daß er ihnen nicht auf einmal die ganze christliche Wahrheit predigen konnte. Deswegen entschloß er sich zu einem ersten pädagogischen Schritt und beschränkte sich darauf, sie vom Götzendienst zum Monotheismus zu führen. Das gelang ihm mit Hilfe des Korans, in dem die wichtigsten Wahrheiten immerhin andeutungsweise beschrieben sind. ${ }^{29}$ )

Der Prophet hatte also doch seine guten Seiten, und der Koran ist nicht einfach ein Teufelswerk, sondern ein Schriftstück voller Ambivalenz. Was nun aber das Islam-Bild des Cusanus angeht, so kann man insgesamt nur feststellen, daß es viele Schattierungen aufweist und großen Spielraum für weitere Interpretationen enthält. Insofern wirkt es vielleicht wenig systematisch. Doch genau das dürfte Nikolaus willkommen gewesen sein. Denn so erhielt er die Möglichkeit, im weiteren Verlauf seiner Schrift mit den diversen Aspekten zu spielen und dem Islam je nach Zusammenhang positive oder negative Seiten abzugewinnen.

Außerdem darf man bei aller Mehrdeutigkeit nicht übersehen, daß sämtlichen Überlegungen ein ganz bestimmter und letzten Endes entscheidender Umkehrschluß zugrundeliegt. Er lautet: „Wenn daher im Koran etwas schön, wahr und klar ist, so rührt das notwendigerweise vom strahlenden Licht des Evangeliums her" ${ }^{30}$ ) Das bedeutet: Was auch immer der Islam uns bietet - alles Gute an ihm kann jedenfalls nur ein Abglanz des Christentums sein. Denn Muhammad war ja schließlich eine Zeitlang ein Anhänger Jesu, so daß es nur naheliegt, wenn die Spuren dieser Begegnung noch heute in seinem Buch und in seiner Religion zu finden sind.

${ }^{28}$ ) Cribratio I 1, N. 22,16-23,11; vgl. auch I 4, N. 28,10-13.

${ }^{29}$ ) Cribratio II 12, N. 119,1-120,10; vgl. auch I 6, N. 40 und I 17, N. 74.

${ }^{30}$ ) Cribratio I 6, N. 41,1-2: Hinc si quid pulchri, veri et clari in Alkorano reperitur, necesse est, quod sit radius lucidissimi evangelii. 
Die Folgerungen, die Cusanus aus diesem Prinzip gezogen hat, sind bekannt und wurden schon mehrfach ausführlich beschrieben. ${ }^{31}$ ) Ihr Charakteristikum besteht darin, daß er meint, Hinweise auf alle wichtigen christlichen Dogmen im Koran finden zu können. Die Gottessohnschaft $\mathrm{Jesu}^{32}$ ) glaubt er dort zu entdecken, den Kreuzestod ${ }^{33}$ ) und die Auferstehung..$^{34}$ ) Und selbst die Trinität, die der Islam ausdrücklich bekämpft hat, scheint ihm in manchen Passagen des Korans vorausgesetzt zu sein. ${ }^{35}$ )

All das kann einen Muslim nur irritieren, und er wüßte an vielen Stellen der Cribratio Alkorani gar nicht, daß von seinem eigenen heiligen Buch die Rede ist. Aber auch wenn Cusanus damit grundsätzlich den Sinn des Korans verfehlt, muß man ihm doch zugestehen, daß seine Erklärungen im Einzelfall unterschiedlich zu beurteilen sind. Manchmal enthält eine Sure durchaus christliche Reminiszenzen, so etwa, wenn von Jesu Geburt aus der Jungfrau Maria berichtet wird. Hier ist es berechtigt, ja, von der Sache her sogar erhellend, wenn man auf den Zusammenhang mit dem Evangelium hinweist. ${ }^{36}$ ) Indessen: Bei solchen bekannten, aber doch sehr äußerlichen Übereinstimmungen zwischen beiden Texten bleibt der Kardinal nicht stehen. Für ihn zählt allein die Idee, daß der Koran in nuce die ganze christliche Botschaft enthalten soll. Sie meint er Schritt für Schritt in der Cribratio zu beweisen. Und um das zu erreichen, deutet er den muslimischen Text großzügig um, oder er mißversteht ihn ganz einfach, nämlich dann, wenn er von einem Übersetzungsfehler in der ihm vorliegenden lateinischen Version des Korans irregeführt wird.

Um beide Arten der Verfälschung kurz zu demonstrieren: Eine Umdeutung liegt beispielsweise vor, wenn Cusanus den Begriff „Wort Gottes“ (kalimat Allāh) im Koran aufgreift und behauptet, daß damit der Logos im

$\left.{ }^{31}\right)$ Burgevin, Cribratio, S. 68 ff.; Hagemann, Kur’ān, S. 117 ff. und ders., Nikolaus von Kues im Gespräch, S. $14 \mathrm{ff}$; vgl. auch Biechler, Nicholas of Cusa, S. $55 \mathrm{f}$.

32) Cribratio I 10-17, N. 54-74.

${ }^{33}$ ) Cribratio II 12-13, N. 115-124.

$\left.{ }^{34}\right)$ Cribratio II 14-15, N. 125-132.

35) Cribratio II 5-11, N. 99-114.

${ }^{36}$ ) Sure 21/91; weitere ausführliche Stellen zu Maria in Sure 19/16 ff. sowie 3/ 42 ff.; zum Thema allgemein A. Schall, Die Sichtung des Christlichen im Koran, in: MFCG 9/1971/76-85; L. Hagemann, Christentum und Christen im Denken zeitgenössischer Muslime, Altenberge 1986, S. $15 \mathrm{ff}$. mit Hinweisen auf die umfangreiche ältere Literatur. 
christlichen Sinne gemeint sei. ${ }^{37}$ ) Ein schlichtes Mißverständnis ist es dagegen, wie er mit Sure 2 Vers 129 umgeht. Dort heißt es ursprünglich im arabischen Text: „Und laß, Herr, unter ihnen einen Gesandten aus ihren eigenen Reihen auftreten . . . ${ }^{\star 38}$ ) In der lateinischen Fassung ist das Wort für Gesandter ( $r a s u \bar{l}$ ) durch mediator, also Vermittler, ersetzt. ${ }^{39}$ ) Und daraus schließt dann der Kardinal, daß auch der Islam die Vorstellung einer Vermittlung zwischen Gott und den Menschen, genauer gesagt, einer Erlösung durch Jesus Christus anerkenne. ${ }^{40}$ )

\section{V}

Was hat all das noch mit einem Gespräch zwischen den Religionen zu tun? Und, so stellt sich abschließend die Frage, kann Nikolaus von Kues, der uns nachdrücklich als Ökumeniker angepriesen wurde, wirklich eine Anregung für den Dialog mit dem Islam sein?

Man ist versucht, die Frage rundum zu verneinen. Denn auf den ersten Blick enthält seine Cribratio in der Tat nur Züge, die einer Begegnung zwi-

${ }^{37}$ ) Cribratio I 12-13, N. 59-62, wobei Cusanus hier nur den Darlegungen früherer christlicher Autoren folgt; die christliche Deutung des koranischen kalimat Allāh dürfte im übrigen das Thema sein; das am häufigsten in der Sekundärliteratur zur Cribratio diskutiert worden ist: Hölscher, Nikolaus von Cues, S. 270; Rescher, Nicholas of Cusa, S. 144; Burgevin, Cribratio, S. 41 f.; Haubst, Die Wege der christologischen manuductio, in: MFCG 16/1984/164 ff., hier: $167 \mathrm{u} .171$; Gandillac, Das Ziel der una religio in varietate rituum, in: MFCG 16\%1984/192 ff., hier 202; Kremer, Die Hinführung, in: MFCG 16/1984/126ff., hier: 153 ff.; Hagemann, Kur’ān, S. 123 ff. und ders., Nikolaus von Kues im Gespräch, S. 14 ff.; vgl. auch A. Falaturi und C. Schedl, in: MFCG 16/1984/270 ff. - Cusanus hat daneben weitere koranische Termini umgedeutet, so etwa die Vorstellung, Gott besitze einen „Geist“ (rūḥ); vgl. Cribratio I 18, N. 75-76; dazu Hagemann; Kur’ān, S. 130 ff.

${ }^{38)}$ rabbañā wa-bcat fihim rasūlan minhưm ...

${ }^{39}$ ) Creator optime . . . nostrae prolis mediatorem prophetam excita . . .; zitiert bei Hagemann, Kur’ān, S. 137.

40) Cribratio III 14, N. 210. Ein weiterer folgenschwerer Übersetzungsfehler liegt Cusanus' Annahme zügrunde, Christus werde im Koran als facies omnium gentium bezeichnet: vgl. Cribratio I 19, N. 77-80 und dazu Anawati, Nicolas de Cues, S. 162; Busse, in: MFCG 16/1984/186; Hagemann, Kur’ān, S. 133 ff. und ders., Nikolaus von Kues im Gespräch, S. 18f. - Auch zahlreiche andere Ungenauigkeiten in der lateinischen Fassung des Korans und daraus folgende Mißverständnisse bei Cusanus machen Hagemann/Glei in den Anmerkungen zu ihrer zweisprachigen Ausgabe der Cribratio aúfmerksam, z. B. in Anm. 73, 110, 202 und 203 zum zweiten Buch. 
schen den Religionen abträglich sind. Dabei ist die suggestive, mitunter beiBende Polemik gar nicht einmal das Störendste. Sie darf man getrost als ein Ergebnis des Zeitalters auffassen, zumal noch mancher spätere Autor, wie etwa Luther ${ }^{4}$ !), sehr viel unsanfter mit dem Islam umgegangen ist.

Bedenklicher wirkt dagegen schon, daß Cusanus, der den Koran erklären wollte, dessen Wortlaut häufig gar nicht verstand. Hier liegt das Grundproblem darin, daß er den Text nicht in der Originalsprache lesen konnte. Aber es wird zusätzlich dadurch verschärft, daß er sich über die Komplikationen, die aus diesem Umstand folgen können, keine Rechenschaft abgelegt hat.

Die letzten Endes größte Schwierigkeit der Cribratio Alkorani ist indessen noch eine andere. Sie besteht, pointiert gesprochen, darin, daß Cusanus die heilige Schrift der Muslime gar nicht verstehen will. Denn er geht zwar in seiner Deutung über die frühere mittelalterliche Polemik hinaus und sucht sowohl am Koran als auch am Propheten Muhammad Züge, die er als positiv und der Anknüpfung wert erachten möchte. Allein: Positiv, „schön, wahr und klar“42) heißt bei Cusanus immer "christlich“. Und dementsprechend fragt er gar nicht lange, wie denn die islamische Exegese den Koran versteht, sondern interpretiert den ganzen Text selbst, und das mit dem Anspruch, damit auch den Muslimen den eigentlichen Sinn ihres heiligen Buches zu erschließen. Hier kann man schwerlich von dem „großartigen theologischen Bemühen des Nikolaus von Kues um eine . . . manuductio ad Christum sowie um einen Brückenschlag . . . ${ }^{43}$ ) sprechen, wie dies gelegentlich in der Cusanus-Forschung geschehen ist. Es handelt sich wohl eher um eine subtile Form der Mißachtung, die den rüden Tonfall älterer Polemiken des Mittelalters durch die sanfte Stimme der Vereinnahmung ersetzt.

Aber bedeutet diese Kritik nun wirklich, daß die vielfältigen Überlegungen der. Cribratio Alkorani keinen einzigen Ansatżpunkt für einen Dialog bieten? Oder findet sich vielleicht doch ein Aspekt, der sich für das sensible, auf jede Anregung angewiesene Gespräch zwischen den Religionen fruchtbar machen ließe? Kehren wir noch einmal zu den ersten Gedanken des Cusanus über den Islam zurück. Dort sagte er, daß alle Menschen nach

$\left.{ }^{41}\right)$ W. Nijenhuis, Luther en de Islam, in: Nederlands Theologische Tijdschrift 33/1979/195-211; N. Heutger, Luthers Stellung zum Islam, in: Zeitschrift für Religions- und Geistesgeschichte 1/1983/359-362; L. Hagemann, Martin Luther und der Islam, Altenberge 1983 (Christlich-Islamisches Institut. Abhandlungen. 2); P. Schwarzenau, Martin Luthers Türkentraktate von 1529 mit Rückblick auf das Lutherjahr 1983, in: Aktuelle Fragen 1/1984/4-13.

42) Vgl. oben Anm. 30.

${ }^{43}$ ) Haubst, in: MFCG 9/1971/87 Anm. 17. 
einem höchsten Gut, das sie Gott nënen, streben. Und es heißt weiter, daß Gott von keinem Menschen wirklich erfaßt werden kann. Damit hatte Cusanus für einen Moment jene zwei Konzepte aufleùchten lassen, für die er in der Geschichte der Philosophie berühmt geworden ist: die Idee der docta ignorantia und den Gedanken der coincidentia oppositorum. Sie besagen, in aller Kürze formuliert: Der Mensch ist außerstande, etwas in seiner ganzen Resllität zu erkennen; und das gilt insbesondere für Gott, der als die unendliche, unableitbare Einheit alles Gegensätzlichen prinzipiell unfaßbar (inattingibilis) ist. $^{44}$ )

Wollte man diese Gedanken konsequent auf den Bereich der Religionen anwenden, so könnten sie dụrchaus eine Grundlage für den Dialog bilden. Denn sie vereinen den höchsten Respekt vor dem Göttlichen mit der Mahnung zu gelehrten menschlichen Bescheidenheit. Ein Gott, der als Einheit aller Gegensätze gedacht wird, kann auch die Verschiedenheit der Religionen zulassen. Und ein Gelehrter, der die Grenzen seines eigenen Wissens kennt, wird in der Beurteilung anderer Vorstellungen zurückhaltend sein.

Zugegeben: Eine solche Vorstellung ist für jeden Gläubigen mühsam, und sie dürfte sogar für einen Muslim unserer Tage ganz besonders schwierig sein..$^{45}$ ) Außerdem könnte man sehr wohl einwenden, daß Cusanus weder die docta ignorantia noch die coincidentia oppositorum in dem hier vorgeschlagenen Sinne verstanden haben wollte. ${ }^{46}$ ) Indessen: Mühe bereitet eine Verständigung immer, und die Tatsache, daß der Kardinal diese Auslegung nicht teilte, muß für uns nicht bindend sein. Denn man weiß ja, daß viele Ideen fern von ihrem Ursprung eine ganz eigene Fruchtbarkeit entfaltet haben. Und so könnten auch diese beiden Gedanken des Cusanus den Dialog zwischen den Religionen bereichern - obwohl ihm selbst dergleichen mit seiner "Sichtung des Korans" nicht gelungen ist.

${ }^{44}$ ) Immer noch eindringlich ist die Darstellung von J. Hirschberger, Geschichte der Philosophie, I, Freiburg $1976^{14}$, S. $569 \mathrm{ff}$; vgl. zur Einführung K. Flasch, Das philosophische Denken im Mittelalter. Von Augústin zu Machiavelli, Stuttgart 1987, S. 540 ff.; aus der unübersehbaren Literatur zur Koinzidenz-Lehre seien nur genannt K. Flasch, Die Metaphysik des Einen bei Nikolaus von Kues, Leiden 1973, S. 155 ff. und W. Beierwaltes, Identität und Differenz als Prinzip cusanischen Denkens, in: ders., Identität und Differenz, Frankfurt a.M. 1980. S: $105 \mathrm{ff}$.

${ }^{45}$ ) Für die muslimische Haltung gegenüber dem Christentum vgl. aüch $\tilde{G}$. $C$. Anawati, Polémique, apologie et dialogue islamo-chrétiens. Positions classiques médiévales et positions contemporaines, in: Euntes Docete 22/1969/375-451 sowie L. Hagemann, Christentum und Christen (vgl. Anm. 36).

${ }^{46}$ ) Burgevin, Cribratio, S. 116 weist zurecht darauf hin, daß das Prinzip der coincidentia oppositorum in der Auseinandersetzung mit dem Islam keine Anwendung findet. Das gilt auch für die Schrift De pace fidei, in der es Heinemann, Einheit, S. $118 \mathrm{zu}$ Unrecht vermutet. 